\title{
Formaçáo do terapeuta ocupacional para o trabalho na Atenção Primária à Saúde (APS): contribuiçóes para o debate
}

\author{
Fátima Corrêa Oliver ${ }^{a}$, Adriana Pimentel ${ }^{b}$, \\ Lúcia da Rocha Uchôa-Figueiredo ${ }^{c}$, Stella Maris Nicolau ${ }^{\mathrm{d}}$
}

\begin{abstract}
aDoutora em Saúde Pública, Faculdade de Saúde Pública, Universidade de São Paulo - USP, Docente do Departamento de Fisioterapia, Fonoaudiologia e Terapia Ocupacional, Faculdade de Medicina, Universidade de São Paulo - USP, São Paulo, SP, Brasil

'Doutora em Saúde Pública, Instituto de Saúde Coletiva, Docente do Instituto de Humanidades, Artes e Ciências Professor Milton Santos, Universidade Federal da Bahia - UFBA, Salvador, BA, Brasil 'Doutora em Ciências, Programa de Psicologia, Faculdade de Filosofia, Ciências e Letras de Ribeirão Preto, Universidade de São Paulo - USP, Docente do Departamento de Gestão e Cuidados em Saúde, Universidade Federal de São Paulo - UNIFESP, Campus Baixada Santista, Santos, SP, Brasil dDoutora em Ciências, Programa de Medicina Preventiva, Faculdade de Medicina, Universidade de São Paulo - USP, Docente do Departamento de Terapia Ocupacional, Centro de Ciências Biológicas e da Saúde, Universidade Federal de São Carlos - UFSCar, São Carlos, SP, Brasil
\end{abstract}

\begin{abstract}
Resumo: As autoras apresentam os debates do grupo de trabalho sobre Formação do Terapeuta Ocupacional para a Atenção Primária à Saúde (APS) realizados durante o I Seminário Nacional de Terapia Ocupacional em APS, desenvolvido durante o XII Congresso Brasileiro e IX Congreso Latinoamericano de Terapia Ocupacional, em outubro de 2011 (São Paulo, SP). Também situam o campo da formação profissional para a APS no contexto do Sistema Único de Saúde (SUS) e destacam sua importância para a formação de terapeutas ocupacionais. São apresentados aspectos relevantes das Diretrizes Curriculares Nacionais específicas nas proposições de formação de profissionais para esse nível assistencial, também colocadas pela Política de Reorientação da Formação Profissional em Saúde (Pró-Saúde), bem como as experiências de formação nesse nível assistencial desenvolvidas pelas instituições de ensino de origem das autoras. Conclui-se que o ensino de graduação nesse nível assistencial possibilita contato estreito de estudantes e docentes com as demandas e necessidades de saúde, processo saúde-doença e seus determinantes em cenários mais próximos da vida cotidiana das pessoas acompanhadas e dos serviços. O ensino de Terapia Ocupacional na APS também possibilita a discussão sobre suas contribuições na promoção da saúde e prevenção de doenças, no diagnóstico precoce, tratamento e reabilitação, o que pode facilitar o acesso de populações antes não consideradas prioritárias na APS. Este seminário foi um importante momento de discussão dos desafios da vinculação da profissão ao SUS.
\end{abstract}

Palavras-chave: Terapia Ocupacional, Ensino, Atenção Primária à Saúde, Formação de Recursos Humanos, Currículo, Serviços de Integração Docente-assistencial.

\section{Training of occupational therapists for Primary Health Care (PHC): contributions to the debate}

\begin{abstract}
Herein presented are the topics debated at the research group meeting on Training of Occupational Therapists for Primary Health Care (PHC) that took place during the First National Seminar on Occupational Therapy in PHC, at the XII Brazilian Congress and IX Latin American Congress of Occupational Therapy in October 2011 in Sao Paulo, Brazil. In addition, the field of vocational training for PHC is situated within the
\end{abstract}


context of the Unified Health System (UHS) and its importance for the training of occupational therapists. We present relevant aspects of specific National Curriculum Guidelines suggested for the professional training at this level of care, which have also been addressed by the Reorientation of Vocational Training in Health Policy (PRO Health). Experiences gained by the authors' educational institutions when training at this level of care are also presented. It was possible to conclude that undergraduate studies at this level of care enables students and teachers to come into close contact with health demands and needs, health-disease process and its determinants, within a scenario closer to the everyday life of the people being cared and the service they receive. The teaching of Occupational Therapy (OT) at PHC further allows the debate of its contributions in promoting health and preventing disease, early diagnosis, treatment and rehabilitation, which may facilitate the access to populations previously not considered priority by the PHC. This seminar was important for discussing the challenges of linking the OT profession to the UHS.

Keywords: Occupational Therapy, Teaching, Primary Health Care, Human Resources Formation, Curriculum, Teaching Care Integration Services.

\section{Introdução}

No Brasil, desde a década de 1960 têm-se implementado propostas de integraçáo entre o ensino de graduação em saúde e os serviços assistenciais. A necessidade de ensino das concepçóes e práticas relacionadas à prevenção de doenças e promoção da saúde, assim como de formação de profissionais com uma visão mais integral da assistência e melhor preparados para lidar com os desafios da atenção em saúde nos serviços públicos estimulou a criação de departamentos especializados em instituições de ensino superior, a proposição de currículos mínimos e o incentivo à criação de associaçóes de educação e ensino das profissóes da saúde (MARSIGLIA, 1995).

A autora destaca diferentes iniciativas associadas a propostas de mudanças no sistema de saúde empreendidas desde 1977, com o Programa de Interiorização das Açóes de Saúde e Saneamento (PIASS), até a constituiçáo do Sistema Único de Saúde (SUS), quando se estruturaram articulaçóes interinstitucionais para a formação do ensino em saúde no âmbito dos serviços. Já em 1979 se recomendava que as secretarias de saúde dos estados assumissem a articulação entre os sistemas formadores e de prestação de assistência para efetiva integração docente-assistencial.

Foram propostas que assumiram diferentes configuraçôes e que incluíram, desde os anos 1980, os ministérios da Educação e da Saúde, a Organização Panamericana da Saúde (OPAS) e a Fundação W. K. Kellogg, que se desdobraram na Rede de Integração Docente Assistencial (IDA - MEC/SESU) para promover a integração da universidade aos serviços públicos existentes. Posteriormente, em 1985, pesquisadores e profissionais mobilizados para a qualificação da educação profissional em saúde criaram a Rede Unida, que desde seu início é um importante interlocutor nesse âmbito (REDE..., 2012).

Com a constituição de 1988 e o advento do SUS, o setor saúde passa a ser ordenador da formação profissional, a partir de iniciativas para mudanças no ensino que contribuiriam para a construção de um novo paradigma de saúde, com uma concepção abrangente e integradora, em oposição à uma visão reducionista e curativista (CAMPOS, 2003).

Ainda na década de 1990 se desenharam outras propostas de integração ensino, serviços e comunidade, como a proposta UNI (Universidade Integrada à Comunidade) com estruturas de caráter tripartite entre universidade, serviços e população (MARSIGLIA, 1995).

As diferentes iniciativas e movimentos relacionados à formação profissional foram rediscutidos entre o Ministério da Educação e representantes das áreas profissionais para organização das Diretrizes Curriculares Nacionais (DCN) propostas entre 1998 e 2001. Tais diretrizes apontavam a necessidade de formação geral e específica de profissionais aptos a trabalhar no contexto do SUS, a partir das realidades locais, em serviços de diferentes níveis de atenção, tanto de atenção primária à saúde (APS) como nos serviços especializados. Discutia-se também a importância da incorporação de metodologias de ensino compatíveis a uma formação crítica e participativa (HADDAD et al., 2010).

Concomitantemente à discussão e implantação das DCN se ampliariam as experiências de APS via Programa de Saúde da Família (PSF) e a formação realizada pelos Polos de Formaçáo, Capacitação e Educação Continuada para a Saúde da Família, 
entre 1998 e 2002, articulando Ministério da Saúde, secretarias estaduais e municipais de saúde.

No Brasil, as expressões Atenção Primária à Saúde e Atenção Básica à Saúde são empregadas ora como sinônimas, ora em contraposição, sendo que a primeira afilia-se à terminologia internacional, que para alguns remete a programas seletivos impostos aos países pobres por políticas neoliberais. Os documentos oficiais brasileiros optam pelo termo atenção básica ao deter-se nos mecanismos financeiros e operacionais do SUS, mas usam os dois termos na perspectiva de unidades locais ou níveis de assistência (GIL, 2006; MELLO; FONTANELLA; PIVA, 2009; BRASIL, 2011b).

A proposição da APS de contextualizar suas açóes à realidade local das pessoas e grupos exige uma prática mais reflexiva dos profissionais no lidar com situaçóes não capturadas pela lógica biomédica (MENDES, 1996). Dessa forma faz-se necessário qualificar profissionais de saúde que atuam na atenção básica e fortalecer as instituiçóes para a reorganização do processo de trabalho, principalmente no âmbito da Saúde da Família, possibilitando assim uma abordagem integral, integrada e resolutiva (BRASIL, 2001).

Nesse sentido, são propostas ações específicas para mudanças curriculares nos cursos de medicina, quando, para a articulação das instituiçóes de ensino à rede de atenção do SUS (BRASIL, 2002b), é lançado, em 2002, o Programa de Incentivos às Mudanças Curriculares dos Cursos de Medicina (Promed).

Em 2003, a formação profissional para o SUS ganha maior institucionalidade com a criação da Secretaria de Gestão do Trabalho e da Educação na Saúde (SEGTS), que contribuiu para o desenho da formação profissional propondo a criação de Polos de Educação Permanente em Saúde, com participação das secretarias de saúde, entre 2003 e 2006 (CECCIM, 2008).

No ano seguinte houve a proposição de cooperação técnica e operacional e de apoio institucional entre as instituições de ensino e os serviços de saúde, como o Aprender SUS e o Programa Vivências e Estágios na realidade do Sistema Único de Saúde (ver SUS). Esse último oferece a estudantes universitários vivências no Sistema Único de Saúde com duração de 15 a 20 dias (CECCIM, 2008).

Somam-se a essas propostas a ação coletiva entre o Ministério da Saúde e as associaçóes de ensino das profissóes de saúde para criação do Fórum Nacional de Educação das Profissões na Área da Saúde (FNEPAS), com participação da Rede Nacional de Ensino e Pesquisa em Terapia Ocupacional (RENETO). Esse fórum mobilizou profissionais em diferentes propostas de fortalecimento da formação em atenção básica com eventos regionais, gerais e por áreas profissionais, incluindo a terapia ocupacional.

Outra proposta foi a criação, em 2005, do Programa Nacional de Reorientação da Formação Profissional em Saúde (Pró-Saúde), articulação entre os ministérios da Saúde e da Educação, que amplia o Promed e integra os cursos de Enfermagem, Medicina e Odontologia, programa que foi ampliado para os 14 cursos de graduação da área da saúde (BRASIL, $2007 \mathrm{a}, \mathrm{b})$. Esses programas procuram desenvolver o ensino em cenários de prática nos serviços do SUS com ênfase na reorientação teórica, considerando-se os determinantes do processo saúde-doença e as necessidades de cuidado, na orientação pedagógica, com propostas de modificação das metodologias para favorecer o ensino crítico e participativo entre serviços e universidade, oferecendo recursos financeiros para qualificar a infraestrutura dos cenários de prática nos serviços e a de ensino nas universidades participantes.

O Pró-Saúde promove uma educação mais humanista, socialmente comprometida, orientada por visão ampliada de saúde, com foco na APS, compreendida, conforme Oliveira (2008), como formação reordenadora da atenção, considerando o trabalho interdisciplinar, a responsabilidade sanitária por um território, a construção de vínculos entre serviço e usuário, a abordagem do sujeito, da família e do contexto, a busca da integralidade e da reformulação de saberes e de práticas.

O fio condutor do Pró-Saúde é a integração ensinoserviço-comunidade, impondo a função de formação (em que a aprendizagem resulta do conhecimento multidimensional e interdisciplinar articulado ao processo de prestação de serviço), a responsabilidade pela qualificação dos trabalhadores e a organizaçáo do trabalho em saúde, contribuindo assim para a formação de novos perfis profissionais capazes de responder à realidade da saúde da população (HADDAD, 2011).

Outra estratégia aliada ao Pró-Saúde foi o Programa de Educação pelo Trabalho em Saúde (PET-Saúde), iniciativa dos ministérios da Saúde e da Educação (BRASIL, 2008b, 2010), que disponibiliza bolsas para tutores, preceptores (profissionais dos serviços) e estudantes de graduação da área da saúde, em grupos tutoriais como: Saúde da Família; Vigilância em Saúde; e Saúde Mental, também com participação de cursos de Terapia Ocupacional. Essas duas iniciativas - PET e Pró-Saúde - compóem um movimento para 
empreender mudanças em um sistema de ensino e pesquisa altamente especializado no país.

A formação de profissionais de saúde demanda discussôes sobre o planejamento de políticas de qualificação profissional, sobre a estrutura universitária e as necessidades de atenção, como, também, sobre as demandas da graduação de profissionais em diferentes campos e regiôes do país, associadas às possibilidades de absorção pelo SUS (HADDAD et al., 2010).

\section{O ensino da APS na formação de terapeutas ocupacionais frente às demandas do SUS}

Em que pese o pequeno número de profissionais e de cursos de graduaçáo no país, os docentes de Terapia Ocupacional e as associaçóes da categoria sempre compuseram nas iniciativas dos ministérios da Educação e da Saúde na discussão da formação profissional, para a implantaçáo dos currículos mínimos, das diretrizes curriculares nacionais, no fórum de associações de ensino e nas propostas de reformulação da formação profissional apresentadas anteriormente.

O debate e apresentação das DCN foram integrados por docentes da área das diferentes regiôes do país que contavam com cursos de graduação. As DCN preconizam que o egresso do curso tenha uma formação generalista, reflexiva e crítica, no plano clínico-terapêutico assim como no campo preventivo das práticas de Terapia Ocupacional e que atue em diferentes níveis de complexidade do sistema de saúde vigente no país, em cada estágio da formação (BRASIL, 2002a).

As diretrizes valorizam a construção de currículos flexíveis e com uma concepção ampliada de saúde que incentivem a autonomia dos estudantes e favoreçam o contato com a população e a realidade dos serviços aos quais o profissional estará vinculado após a graduação. Nesse sentido é importante que o estudante experimente a prática profissional em diferentes equipamentos sociais e de saúde, tais como unidades básicas de saúde e comunidades. Mais que isso, vivencie práticas que exijam dele novas formas de atuação e interlocuçáo com o outro, por exemplo, quando dificuldades e possibilidades surgem dos diferentes interesses e necessidades das pessoas que vivem em contexto de pobreza. Para isso, nas diretrizes está previsto que os currículos orientem a formação para a compreensão, interpretaçáo e proteção de diferentes realidades socioculturais.

Vinculados a essa perspectiva da implantação das DCN nas diferentes áreas da saúde, os profissionais e docentes também têm participado das discussóes e debates propostos pelo FNEPAS, em diferentes oficinas regionais e locais sobre os desafios da implementação da formação profissional e interprofissional para construção e defesa do SUS, com ênfase nos aportes para a APS.

Vale destacar a organização, em 2007, pelo Conselho Federal de Fisioterapia e Terapia Ocupacional, da oficina intitulada Equidade, Ética e Direito à Saúde: Formação e Ação da Terapia Ocupacional no V Congresso de Ciências Sociais e Humanas em Saúde, ocorrido em Salvador, Bahia, durante a qual se discutiu a formaçáo permanente do terapeuta ocupacional para atuação no SUS. Um dos grupos de discussão dedicou-se ao mapeamento e descrição da formação do terapeuta ocupacional, constatando: a necessidade de uma reorganização curricular com ênfase na atenção básica; a dificuldade de articular estágios em virtude da ausência de terapeutas ocupacionais nos serviços; a necessidade de maior envolvimento dos profissionais com as demandas regionais e de maior articulaçáo entre universidades e serviços.

Nesse grupo foi considerada a heterogeneidade de implementação das DNC nos diferentes cursos, a necessidade de fortalecimento dos vínculos entre serviços e escolas e de se conhecer as demandas regionais de saúde para orientar a formação dos estudantes, a necessidade de implantação de Residências Multiprofissionais em APS e de superação do modelo biomédico na formação do terapeuta ocupacional, assim como a necessidade de se organizar eventos que fortaleçam a discussão sobre a formação e atuação dos terapeutas ocupacionais no SUS (CONSELHO..., 2007).

As instituiçôes de ensino em Terapia Ocupacional organizaram-se para participar das propostas de reorientaçáo da formaçáo como, por exemplo, das Residências Profissionais e Multiprofissionais em Saúde e do Pró-Saúde. Esses encontros contaram com a participação de sete cursos de Terapia Ocupacional, em um universo de 49 escolas de graduação em atividade no país (BRASIL, 2011a; PALM, 2012).

Embora em relação a outras categorias exista menor proporção de terapeutas ocupacionais no país, a presença da área no PET-Saúde revela o empenho dos cursos em participar dessa política que integra educação e trabalho, com maior relevância para os projetos ligados ao PET-Saúde Mental, conforme 
Tabela 1. Soares (2008) considera essa participação desafiadora por ampliar conceitos norteadores para as áreas profissionais e, em especial, para a terapia ocupacional, por demonstrar que esta tem afinidades com objetivos, propostas e abordagens da atenção básica, como também por suas contribuições para a Estratégia de Saúde da Família e para a constituição dos Núcleos de Apoio à Saúde da Família - NASF, que contam com a inserção do profissional terapeuta ocupacional (BRASIL, 2008a).

No entanto, para o conjunto dos profissionais $\mathrm{da}$ área observa-se que ainda é pequeno, mas crescente, o interesse pelo campo de atividades assistenciais relacionadas à APS, conforme apontou Carrasco-Bassi (2012), em estudo sobre os trabalhos apresentados nos congressos brasileiros de terapia ocupacional, no período entre 1999 e 2011. A autora identificou que entre 3.755 trabalhos apresentados nesses eventos apenas 191 (5\%) tratavam do tema. E mesmo nos congressos de 2009 e 2011, posteriores à criação dos NASF, somente 5,8 e $6,2 \%$ dos trabalhos apresentados, respectivamente, tratavam de temas relacionados à APS.

A autora também observou que entre 2000 e 2011 foram publicados 21 artigos sobre o tema na Revista de Terapia Ocupacional da USP e nos Cadernos de Terapia Ocupacional da UFSCar, a quase totalidade deles de autoria de docentes, o que demonstra uma preocupação das instituiçốes de ensino no tratamento desse tema em suas atividades universitárias.

\section{Contribuições do Grupo} de trabalho Formação de Terapeutas Ocupacionais para a APS no XII

\section{Congresso Brasileiro Terapia Ocupacional (XII CBTO)}

Durante o XII CBTO realizou-se o I Simpósio de TO na APS e nele o Grupo de Trabalho Formação de Terapeutas Ocupacionais para a APS, com
24 participantes, sendo nove docentes de oito universidades, dez estudantes de graduação de cinco universidades e cinco profissionais de serviços de APS e de assistência social.

O documento preliminar apresentado ao grupo como disparador dos debates aponta o crescimento de trabalhos relacionados à APS no CBTO em 2009 e a importância das políticas indutoras na inserção da Terapia Ocupacional na APS, tais como, o Pró-Saúde, o PET-Saúde, as Residências Profissionais e Multiprofissionais em Saúde. O documento reafirma a importância das DCN (BRASIL, 2002a) nas revisôes e readequaçôes dos projetos pedagógicos dos cursos de graduaçáo em Terapia Ocupacional (PIMENTEL; OLIVER; UCHÔA-FIGUEIREDO, 2012).

Também foram colocadas questões para o debate como: os currículos refletem seus princípios orientadores? As mudanças nos projetos pedagógicos atendem às especificidades da atuação na APS? Os projetos pedagógicos consideram a importância estratégica da APS como reordenadora do modelo assistencial? As adequaçóes curriculares têm promovido mudanças nas práticas dos terapeutas ocupacionais nos serviços? Os componentes curriculares contemplam a interdisciplinaridade, as ações extramuros e em diversos contextos socioculturais?

Alguns representantes das universidades e dos serviços apresentaram suas experiências e constatou-se que a maioria dos cursos têm promovido mudanças curriculares, algumas mais pontuais e outras mais estruturais. Alguns cursos optaram pela inclusão de disciplinas relativas à saúde coletiva e à APS, além da inserção de estudantes em atividades práticas e estágios nos serviços de APS, como relataram a Universidade Federal do Paraná - UFPR e a Universidade de São Paulo - USP-SP. A primeira tem como desafio formar profissionais em uma região com escassez de terapeutas ocupacionais, como é o estado do Paraná, onde a presença desse profissional praticamente inexiste na APS. A segunda contempla a APS há mais de duas décadas e aponta que os

Tabela 1. Abrangência dos programas PET para o ano de 2011.

\begin{tabular}{lcc}
\hline \multicolumn{1}{c}{ Programas - PET } & $\begin{array}{c}\mathbf{N}^{\circ} \text { de projetos } \\
\text { selecionados }\end{array}$ & $\begin{array}{c}\mathbf{N}^{\mathbf{0}} \text { de projetos com } \\
\text { TO selecionados }\end{array}$ \\
\hline PET - Saúde/Saúde da Família 2010/2011 - Edital n ${ }^{\circ}$ 18/2009 & 111 & 14 \\
PET - Saúde/Vigilância em Saúde 2010/2011 - Edital n $7 / 2010$ & 70 & 5 \\
PET - Saúde/Saúde Mental/Crack 2011 - Edital n' 27/2010 & 69 & 11 \\
Total & 250 & 30 \\
\hline
\end{tabular}

Fonte: Brasil (2012). 
egressos dessa formação têm conseguido inserir-se com êxito na APS.

Outros cursos construíram projetos pedagógicos onde a APS é eixo transversal na formação do estudante, como nas experiências de cursos novos da Universidade Federal de Sergipe (UFSE) e da Universidade Federal de São Paulo - Baixada Santista (UNIFESP-BS) e de cursos mais antigos, como o da Universidade Federal de São Carlos (UFSCar) e da Universidade de Fortaleza (Unifor). Na UFSE, os oito cursos de graduação na área da saúde - incluindo o de Terapia Ocupacional - desenvolvem práticas de ensino na comunidade desde o primeiro ano, atuando junto a populaçóes urbanas e rurais em espaços comunitários de territórios adstritos.

A participação no PET-Saúde foi citada pelos cursos da UFSCar, Unifor, Universidade Federal de Minas Gerais (UFMG), UFPR, USP-SP como política que promoveu maior contato dos estudantes de Terapia Ocupacional com alunos de outros cursos e com o cotidiano dos serviços de APS, experiência valorizada pelos presentes na discussão.

Os profissionais apontaram problemas como o impacto nem sempre positivo da presença de muitos estudantes em início de curso nos serviços e a necessidade de os terapeutas ocupacionais serem capacitados para o trabalho na APS como, por exemplo, nos Programas de Residência. Os profissionais preceptores de estudantes de graduação empreendem grandes esforços para conciliar as atividades de ensino e assistência com sua carga horária e reivindicam maior reconhecimento institucional da função de preceptor.

Em suma, houve um consenso de que os cursos vêm gradativamente incorporando a formação na APS, embora com variaçôes na forma e carga horária dedicada ao campo. Ao mesmo tempo, considerou-se a necessidade de ampliar o debate para outros âmbitos da formação como as residências e aprimoramentos, as pós-graduações strito e lato senso, como também de ampliar o apoio à educaçáo permanente dos profissionais que não contaram com formação específica e que demandam continuidade da formação tendo em vista os problemas e desafios colocados pelo desenvolvimento da assistência. Outro ponto levantado abordou a importância da parceria entre universidade e serviços no desenvolvimento de atividades assistenciais e de ensino, pois esse é um processo complexo que implica discussóes e negociaçôes permanentes.

\section{Experiências de formação de terapeutas ocupacionais na Atenção Primária à Saúde: contribuições ao debate}

\subsection{Curso de Terapia Ocupacional da Faculdade de Medicina da Universidade de São Paulo - FMUSP}

Desde 1982 há prioridade para a formação crítica de estudantes articulada às políticas públicas, especialmente às relacionadas às pessoas com deficiências e em sofrimento psíquico. Essa prioridade foi construída por docentes, participantes das reformas Sanitária e Psiquiátrica e de grupos de trabalho para o desenvolvimento de políticas de atenção específicas de secretarias e do Ministério da Saúde. Tais atores atuaram na criação e implementação de serviços alternativos à institucionalizaçáo das pessoas, para a construçáo e exercício de cidadania, movimentos com desdobramentos diferenciados na atenção à saúde mental e às pessoas com deficiências, que repercutiram na formação dos profissionais na FMUSP.

No que diz respeito à APS houve destaque para alternativas de atenção a pessoas com deficiências e idosos. Já em 1985, docentes participavam em grupos de trabalho para criação do Sistema de Atendimento à Saúde e Reabilitação da Pessoa Portadora de Deficiência Física e/ou Sensorial na Secretaria Estadual de Saúde de Sáo Paulo (SES), questionando a centralidade dos serviços especializados de reabilitação no modelo assistencial. Isso resultou em parceria para a realização de projeto piloto de rede regionalizada, em centro de saúde na região norte do município, onde, em 1987 e 1988, estudantes realizavam estágios curriculares acompanhando a construção de tecnologias de cuidado compatíveis com os problemas vivenciados pelas pessoas e famílias.

Posteriormente, em 1989, docentes participaram da elaboração de perfil do profissional para a criação de cerca de 200 cargos de terapeuta ocupacional na Secretaria Municipal de Saúde de São Paulo (SMS), como também dos fóruns para a constituição das políticas assistenciais e rede de serviços, incluindo pesquisas que documentaram esses processos (LOPES, 1999; OLIVER; BARROS; LOPES, 2005; HO; OLIVER, 2005).

Fruto desse trabalho, desde 1992 estabeleceram-se novas parcerias com unidades básicas de saúde (UBS) da região oeste do município de São Paulo, UBS que contavam com terapeuta ocupacional 
contratado, como as de Vila Dalva (1992-1996) e Jardim Boa Vista (desde 2001), esta com Programa de Saúde da Família (PSF), como também parceria com a comunidade do bairro Jardim D'Abril (de 1998 a 2003) e com a UBS São Jorge (desde 2008).

Em geral, as experiências partiram da identificação das pessoas com deficiência, suas necessidades de saúde e reabilitação e da discussão de alternativas assistenciais nos territórios e serviços, para maior visibilidade desse grupo e para implementar processos participativos e de afirmação de direitos (ALMEIDA; TISSI; OLIVER, 2000; OLIVER et al., 2001; NICOLAU; AOKI; OLIVER, 2007), inspirados nos princípios da reabilitação baseada na comunidade. Participaram estudantes das disciplinas Terapia Ocupacional Social (optativa) e Terapia Ocupacional em Deficiência Física (obrigatória).

Paralelamente, entre 1999 e 2008, docente do curso elaborou e assessorou o Projeto Implantação do Programa de Atenção à Saúde da Pessoa Portadora de Deficiência no Programa Saúde da Família - Qualis, parceria entre a SES e a Fundaçáo Zerbini na regiáo leste do município. Essa foi iniciativa pioneira para a atenção em reabilitação no PSF, onde se realizou Estágio Supervisionado em Terapia Ocupacional em Deficiência Física e pesquisas sobre o programa (ROCHA; PAULA; KRETZER, 2004; ROCHA; KRETZER, 2009; ANTUNES; ROCHA, 2011; ROCHA; SOUZA, 2011).

Entre 2001 e 2003 se efetivou outro desenho curricular com um eixo de disciplinas relacionadas aos processos de adoecimento, à análise crítica das políticas de saúde e à constituição da Terapia Ocupacional e das atividades e recursos terapêuticos além das disciplinas dos campos de Deficiência Intelectual, Saúde Mental, Saúde e Trabalho, Saúde da Pessoa com Deficiência, Geriatria e Gerontologia e Terapia Ocupacional Social. Outro eixo de disciplinas de apoio ao conhecimento de alternativas assistenciais junto a pessoas, grupos ou comunidades foi constituído por nove disciplinas optativas eletivas relacionadas aos campos anteriormente mencionados, as Práticas Supervisionadas ( 150 horas cada - do $4^{\circ}$ ao $6^{\circ}$ semestres) e outras nove de Estágios Supervisionados (420 horas cada - do $7^{\circ}$ e $8^{\circ}$ semestres).

Entre as disciplinas vinculadas a atividades da APS estão as de Prática e Estágio Supervisionado em Terapia Ocupacional na Atenção Territorial e Comunitária em Reabilitação, de Estágio Supervisionado em Terapia Ocupacional e Deficiência Física e de Prática e Estágio Supervisionado em Terapia Ocupacional em Geriatria e Gerontologia, essas últimas realizadas nos centros de saúde da Faculdade de Saúde Pública e da Faculdade de Medicina, no Hospital Universitário e no Centro de Convivência e Cooperativa da SMS.
O estudante deve cursar três disciplinas do grupo de nove práticas e outras duas do grupo de nove estágios, que são oferecidas por docentes e terapeutas ocupacionais dos serviços e/ou da universidade, os quais acompanham grupos de até seis alunos em atividades assistenciais e reflexivas nesse âmbito da formação.

As propostas indicadas influenciaram profissionais e gestores de outros municípios na implantação de políticas específicas, na criação do curso de especialização Terapia Ocupacional em Reabilitação, Recursos Tecnológicos e Inclusão Social da Pessoa com Deficiência e em iniciativas de educação permanente para médicos, dentistas, agentes comunitários de saúde e profissionais de reabilitação das equipes dos serviços. Também possibilitaram o desenvolvimento de inúmeros projetos de extensão, de pesquisas de graduação, especialização, mestrado (na linha de pesquisa Intervenção Social e Terapia Ocupacional do Programa de Ciências da Reabilitação - FMUSP) e em editais específicos de agências de fomento (CALDEIRA, 2009; AOKI, 2009; FERREIRA, 2009; VARELA, 2010; LANCMAN; BARROS, 2011; OLIVER et al., 2012).

Desde fevereiro de 2012, as disciplinas de Prática e Estágio Supervisionados em Saúde Mental também se realizam em parceria com dois NASF e UBS de referência, na regiáo oeste do município, compondo as possibilidades de ensino na APS da FMUSP.

O curso também participou, entre 2009 e 2012, dos PET Saúde da Família e Saúde Mental, com outros nove cursos de saúde do campus da capital.

A integração entre ensino e serviços é fruto de articulação interinstitucional marcada pelas periódicas mudanças de política das gestóes (municipal, estadual ou federal) e são experiências que se desenvolvem em meio às contradiçóes da constituição do SUS em São Paulo, maior e mais rico município do país, porém com uma rede de serviços insuficiente e desarticulada para o acompanhamento das demandas de saúde da população.

\subsection{Curso de Terapia Ocupacional da Universidade Federal de São Carlos - UFSCar}

O curso de Terapia Ocupacional da UFSCar foi implantado em 1978 e passou por reformulaçóes curriculares, mas desde o seu início adotou o conceito de saúde associado à vida em sociedade e "[...] a uma compreensão mais integral do ser humano, em seus processos de desenvolvimento e inserção social [...]" (UNIVERSIDADE..., 2007, p. 17).

Com a reformulação de 1984, seguida de adaptaçóes na estrutura curricular nos anos de 1988 
e 1992, a disciplina Noçóes de Saúde Pública, com 60 horas, abordava aspectos históricos e conceituais das políticas de saúde no Brasil e promovia o reconhecimento dos limites e possibilidades do desenho local e da articulação do sistema de saúde no país para o desenvolvimento de propostas de atençấo às pessoas com deficiências e pessoas em sofrimento mental.

Em São Carlos eram escassas as experiências de terapeutas ocupacionais atuando na APS (CARRASCO-BASSI, 2012) e desde o final da década de 1990 essa possibilidade de formação prática era feita nos estágios profissionalizantes optativos de último ano, em outros municípios, por meio de parceria entre o Laboratório METUIA do Departamento de Terapia Ocupacional da UFSCar e o Projeto Qualidade Integral em Saúde - Fundação Zerbini/PSF (Qualis Zerbini), realizado na cidade de São Paulo.

Parte da pesquisa Açóes Básicas de Saúde e a Construção do SUS: Cidadania, Direitos e Políticas Públicas (LOPES et al., 2007), o subprojeto Possibilidades e Limites da Terapia Ocupacional no Programa de Saúde da Família - PSF (LOPES; PALMA, 2005), permitiu a articulação com a equipe de saúde mental do Qualis Zerbini, que contava com terapeuta ocupacional na supervisão de estágios a alunos desse curso até o ano de 2007.

Outro subprojeto daquela pesquisa dedicou-se à atenção às pessoas com deficiência e pessoas em sofrimento mental pela Estratégia de Saúde da Família no município de São Carlos, a partir das açôes dos Agentes Comunitários de Saúde na cidade. Nesse trabalho, alunos de graduação participaram de forma optativa, também da elaboração de uma proposta de formação para os ACS lidarem com esses grupos populacionais (LOPES; FURLAN; BRITO, 2006).

Em 2006, implanta-se na UFSCar o curso de Medicina que adotou metodologias ativas de aprendizagem, com forte inserção na rede municipal de saúde de São Carlos, o que criou um contexto favorável a mudanças curriculares e influenciou a reestruturação do projeto pedagógico do curso de Terapia Ocupacionalda UFSCar, colocado em prática em 2008, com ampliaçáo de 30 para 40 vagas de ingressantes no ano seguinte.

A implantação da Residência Multiprofissional em Saúde da Família, em 2007, o desenvolvimento do Pró-Saúde (2009 a 2011) e do PET-Saúde da Família (2009 a 2012), com a participação do curso de Terapia Ocupacional da UFSCar fomentaram as mudanças curriculares e a vinculação aos serviços de APS no município.
$\mathrm{O}$ atual projeto pedagógico pauta-se por uma concepção de educação socioconstrutivista, com metodologias ativas de aprendizagem - aprendizagem baseada em problemas, problematização de base freireana, experimentação de situações da prática profissional em cenários reais e simulados. Esse projeto está em consonância com as DCN e visa ao desenvolvimento de quatro áreas de competência, a saber: atençáo a indivíduos, grupos e coletivos, gestấo, educação e pesquisa.

As disciplinas básicas e profissionalizantes são substituídas por unidades educacionais integradas, em um total de cinco a cada ano: teórica, prática, recursos e atividades, pesquisa e consultoria orientada. Adota-se também um currículo por competência, compreendida como a capacidade de empregar conhecimentos, atitudes e habilidades de forma efetiva e qualificada para responder aos desafios da prática profissional (UNIVERSIDADE..., 2007).

O eixo da prática tem início no primeiro ano do curso e os estudantes cumprem uma carga horária de 600 horas de atividades nos dois primeiros anos, sendo 200 horas em grupos de reflexáo mediados por docentes e 400 horas nos serviços de APS, onde são acompanhados por preceptores terapeutas ocupacionais da rede municipal de saúde de São Carlos, que mantém uma relaçáo de parceria com a universidade.

Os 80 estudantes são subdivididos entre as sete terapeutas ocupacionais que recentemente se integraram ao trabalho na atenção básica por demandas da parceria universidade e serviços. Os estudantes são distribuídos em dez UBS das cinco regiōes de saúde da cidade, sendo que uma mesma preceptora recebe estudantes em mais de uma unidade de saúde, e esses estudantes permanecem no mesmo serviço por dois anos. Em um futuro próximo há a perspectiva de estágios nos últimos anos do curso na APS.

Nos serviços de saúde, os estudantes realizam atividades de reconhecimento do território e seus recursos, observam diferentes setores e postos de trabalho, para apreenderem como é o trabalho na APS e a articulação dos profissionais na sua realização. Gradativamente engajam-se em tarefas do cuidado às famílias, em grau progressivo de responsabilidade, as quais requerem o desenvolvimento da capacidade de empatia, escuta atenta e qualificada, formação e manejo de vínculo afetivo com a equipe, colegas e usuários, identificação de necessidades de saúde de forma ampliada, participação na construçáo e efetivação de planos de cuidado individual, grupal e coletivo, no âmbito da promoção, prevenção e tratamento. 
$\mathrm{O}$ contato dos estudantes com a equipe e a comunidade é mediado pelo profissional terapeuta ocupacional, que lhes apresenta elementos do campo da saúde coletiva e do núcleo da terapia ocupacional, apoiando a construçáo de planos de cuidado que também envolvam outros profissionais da equipe e açôes intersetoriais no território. Nesse sentido, o preceptor tem um importante papel formador em serviço, o que possibilita aos estudantes um contato estreito com o mundo do trabalho no âmbito do SUS e com um terapeuta ocupacional em uma situaçáo de trabalho real, embora o foco da prática não seja somente o trabalho do terapeuta ocupacional, mas sim o trabalho em APS e o desenvolvimento de habilidades relacionais e de identificação de necessidades de saúde.

Em setembro de 2011, o curso organizou na UFSCar o I Fórum de Terapia Ocupacional Trajetórias e Perspectivas na Atenção Básica no Município de São Carlos, com relatos de experiências e debates.

Destacam-se também trabalhos de iniciação científica (BIANCHI; MALFITANO, 2011; CARVALHO; MATSUKURA, 2011), projetos de extensão (CRUZ; NICOLAU; FARIA, 2011; NUNES; DELLA BARBA, 2010; SILVEIRA; BRITO, 2011) e dissertação de mestrado do Programa de Pós-Graduação em Terapia Ocupacional que aborda as práticas de terapeutas ocupacionais na APS (CARRASCO-BASSI, 2012), além de trabalhos decorrentes dos projetos PET-Saúde em que está inserida a Terapia Ocupacional e que são apresentados nos simpósios anuais da UFSCar desde 2010.

\subsection{A experiência de formação no curso de Terapia Ocupacional da Escola Bahiana de Medicina e Saúde Pública (EBMSP)}

Na busca da construção de um sistema de saúde que priorize as necessidades e demandas mais candentes dos sujeitos sob sua responsabilidade e que seja coerente com o ambiente de vida desses sujeitos é que a APS deve se desenvolver, a fim de constituir-se propulsora de formação e novas práticas profissionais. Como forma de reorientaçáo do modelo de atenção em reabilitação, algumas experiências de ensino e pesquisa orientadas pelas estratégias da Reabilitação Baseada na Comunidade (RBC) e Reabilitaçáo Psicossocial (ALMEIDA; OLIVER, 2001) vêm ocorrendo no país. Foi a partir desses referenciais que desenvolveu a experiência no curso de Terapia Ocupacional da EBMSP, em Salvador.

O curso da EBMSP foi criado em 1968. Em 1998 foi formada a primeira turma após reabertura do curso em 1994. Nesse período já se discutia a necessidade de uma formação mais contextualizada às transformaçóes por que passava o modelo de atenção à saúde em todo o país. Em função disto, em 2005 foi criado o Grupo de Pesquisa Modos de Vida e Territórios Urbanos. Esse grupo teve como finalidade fomentar estudos e práticas dirigidas às populaçóes-alvo dos terapeutas ocupacionais, como pessoas com deficiência (PCD), em sofrimento mental e outros grupos em processo de vulnerabilidade e desfiliação.

A proposta enfatizava estudos sobre o contexto de vida dessas pessoas, suas práticas cotidianas e estratégias de enfrentamento das situaçóes que experimentavam. Em 2006 foi elaborado um projeto de pesquisa para conhecer o perfil das pessoas com deficiência em um bairro da cidade de Salvador, priorizando atividades e níveis de participaçáo dessas pessoas no território e na cidade. Ao mesmo tempo foi realizado um mapeamento territorial georreferenciado do bairro, a fim de conhecer os recursos, equipamentos e atividades desenvolvidas na área de abrangência do estudo, o que proporcionou a criação de um banco de dados que subsidiou pesquisas de discentes e docentes, contribuindo para a elaboraçáo e execução de projetos de extensáo para estudantes dos cursos de Terapia Ocupacional e Fisioterapia. Em 2008 foi criado um campo de estágio curricular visando a formação de estudantes de Terapia Ocupacional em saúde coletiva, especificamente na Atenção Básica (PIMENTEL; COSTA; SOUZA, 2011).

A partir do estudo aprofundado de um bairro foi possível iniciar práticas das disciplinas de Saúde Coletiva e Atenção ao Adulto, projeto de extensão, e a abertura de um campo de estágio no intuito de aproximar os estudantes de um universo desconhecido e, ao mesmo tempo, provocar reflexóes sobre práticas territoriais. Os alunos passavam, então, a experimentar situaçóes reais, com sujeitos em seu espaço de vida e de forma articulada com outros saberes. O estágio implicou duas dimensóes do cuidado: a intervenção clínica e as açôes territoriais.

Assim, a partir do levantamento de pessoas com deficiência e do mapeamento territorial georreferenciado, iniciamos as visitas a pessoas com deficiência no bairro. Devido a predominância de pessoas adultas e idosas em situação de isolamento doméstico e com forte dependência de um cuidador, foi necessário um trabalho no domicílio, junto à família e/ou cuidador, cujo objetivo era minimizar os danos de um processo altamente cronificador. Desse modo, cuidar da alimentaçáo e higiene com maior independência e autonomia, facilitar a comunicação e mobilidade e melhorar a condição de 
vida, também do cuidador, eram açōes prioritárias a serem realizadas pelos estudantes.

Esse momento foi crucial para colocar em prática as açôes territoriais. Essas ações visavam o resgate da participaçáo social e o fortalecimento das redes de suporte social. Assim, foram criados grupos para crianças com e sem deficiência; grupos para os cuidadores das pessoas com deficiência; oficina de artes; um Fórum de Discussão sobre Deficiência; ampliação dos espaços de negociação com a unidade de saúde, escolas, associaçóes de moradores; passeios em espaços públicos da cidade; participação em encontros e eventos sobre deficiência, entre outras açôes.

Esse trabalho de formação tem contribuído para sensibilizar estudantes e profissionais para uma prática diferenciada e coerente com os princípios e diretrizes do SUS. Tem também favorecido a produção de conhecimento em terapia ocupacional no campo da saúde coletiva. Dessa experiência resultaram duas publicaçốes em periódicos da área; participação em eventos científicos locais, regionais, nacionais e internacionais; produção de trabalhos de conclusão de curso; formação científica de dez estudantes - alguns destes dando prosseguimento à formação em Programas de Pós-Graduação em Saúde Coletiva. Além disso, foi possível a contratação de uma terapeuta ocupacional na Unidade de Saúde local para dar continuidade às açôes e projetos até entáo desenvolvidos.

\subsection{A experiência de formação no curso de Terapia Ocupacional da Universidade Federal de São Paulo - Baixada Santista (Unifesp-BS)}

As diversas experiências de integração docenteassistencial iniciadas nos anos 1980 mostram a importância dos cenários de ensino e aprendizagem em atenção primária, especializada e hospitalar na rede de serviços de saúde (FEUERWERKER, 2003). Segundo Ceccim e Carvalho (2005), essas experiências revelaram ainda a necessidade de se ultrapassar os limites das disciplinas e da formação isolada dos profissionais para intervir nas necessidades individuais e coletivas de saúde.

Entendendo que as DCN destacam que a formação deve contemplar o sistema de saúde vigente no país, o trabalho em equipe e a atenção integral, a UNIFESP-BS inovou construindo uma proposta curricular que articula o ensino às necessidades de saúde da população, na tentativa de buscar novos caminhos e referenciais que ampliem a formação dos profissionais de saúde.
O campus foi implantado em 2006, inicialmente com os cursos de graduação em Educação Física, Fisioterapia, Nutrição, Psicologia e Terapia Ocupacional e, a partir de 2009, com o curso de Serviço Social, priorizando desde entáo a educação interprofissional, a formação para o trabalho em equipe e para a integralidade da atenção.

A organização das atividades de ensino se dá em módulos, rompendo com a estrutura tradicional centrada em disciplinas e com a formação isolada de determinado perfil profissional. A estrutura curricular dos cursos está definida em quatro eixos, que aproximam o aluno de uma Prática Específica em Saúde e são direcionados aos estudantes de cada área profissional. Desses, são eixos comuns: O Ser Humano e sua Dimensão Biológica; O Ser Humano em sua Inserçáo Social (IS) e o Trabalho em Saúde (TS). Esses últimos eixos se orientam pelos conhecimentos que seriam necessários para todos os profissionais de saúde e são trabalhados com turmas menores, como se fossem equipes de saúde, com alunos dos vários cursos e docentes de áreas distintas (UNIVERSIDADE..., 2006).

O eixo que trabalha especificamente práticas relativas à saúde e seu cotidiano é o TS, que é um norte centralizador no projeto político-pedagógico do campus e que se desenvolve nos três primeiros anos de graduação, possibilitando aos estudantes uma visão abrangente do processo saúde-doença-cuidado, do trabalho em saúde e do sistema de saúde vigente no país, de maneira a formar profissionais capacitados a ofertar atenção integral e desempenhar um papel ativo nas questóes de saúde em nossa sociedade (UNIVERSIDADE..., 2006).

Desde o primeiro ano da graduação o módulo insere os alunos em atividades que possibilitam o contato com diferentes grupos populacionais e seus problemas de saúde e com movimentos sociais, bem como com as equipes de diferentes serviços e locais em que ocorrem açóes de cuidado à saúde.

A aprendizagem se dá através das vivências, interações, exposição a situaçóes do cotidiano do trabalho em saúde. Os alunos são estimulados a assumir responsabilidades crescentes e a realizarem intervenções e prestações de cuidado. A organização dos módulos é semestral, sendo que em cada um acontecem atividades específicas de ensinoaprendizagem integradas aos serviços de diferentes regióes do município de Santos onde vivem pessoas de maior vulnerabilidade social.

O trabalho em saúde só é possível nesse contexto, pois se dá em função do "trabalho vivo em ato", que acontece no momento do encontro entre o trabalhador e quem demanda atenção (MERHY, 2000; MERHY; FRANCO, 2006). A partir da 
identificação da demanda do usuário, feita pela escuta, se delineiam o trajeto, as intervençôes e os recursos necessários para alcançar o que foi proposto.

Pretende-se, com as atividades desenvolvidas no eixo, contribuir para que o aluno possa: apreender a prática clínica dos profissionais de saúde; desenvolver as capacidades de escuta, de estabelecer vínculo, de dialogar e, assim, valorizar o momento do encontro com o usuário.

No decorrer dos três anos, os módulos de TS abordam as múltiplas dimensôes do processo saúde-doença, as políticas e a organização dos serviços de saúde, as possibilidades de ampliar a prática clínica comum aos diversos profissionais de saúde, a importância de valorizar os referenciais de trabalho de cada profissional, bem como de ampliar as possibilidades de atuação conjunta das várias áreas profissionais e as do trabalho em rede.

Em 2008, a necessidade de melhor entendimento desse processo ensino-aprendizagem possibilitou que docentes participantes da nova proposta, alguns do curso de Terapia Ocupacional, criassem o Laboratório de Estudos e Pesquisas em Formação e Trabalho em Saúde (LEPETS), que desenvolve estudos nesse campo.

Em 2010, o campus da Unifesp-BS também criou o programa de residência integrada multiprofissional em atenção à saúde, em parceria com a Santa Casa de Misericórdia de Santos e a Secretaria Municipal de Saúde de Santos (SMSS). Tanto a primeira como a segunda turmas contaram com 14 residentes de sete áreas distintas: Enfermagem, Farmácia, Fisioterapia, Nutrição, Psicologia, Serviço Social e Terapia Ocupacional. Em 2012 se iniciou a terceira turma, que incluiu Educação Física, com 16 residentes. Na residência se realiza o trabalho em equipes multi e interdisciplinares, na perspectiva da integralidade do cuidado e da construção de um trabalho que transcende os fazeres profissionais individualizados, para maior resolutividade dos serviços de saúde e aumento do vínculo com a população atendida, com aposta na intersetorialidade e nos princípios e políticas do SUS.

Também em 2012 teve início o programa de mestrado profissional - Ensino em Ciências da Saúde -, a partir do programa do campus São Paulo, aprovado em 2008, para formar profissionais e implementar a avaliação crítica de suas ações educativas inovadoras, produzir conhecimento sobre o ensino pela problematização e que estejam aptos para a avaliação contínua e transformadora de suas práticas cotidianas no Ensino em Ciências da Saúde. As linhas de pesquisa do programa são: Avaliação, currículo, docência e formação em saúde; Educação permanente em saúde e Educação em saúde na comunidade. $\mathrm{O}$ curso foi autorizado e reconhecido pela Coordenaçáao de Aperfeiçoamento de Pessoal de Nível Superior (CAPES) com conceito 4.

As atividades do PRO e PET-Saúde (Rede Cegonha e Saúde Mental) terão início no segundo semestre de 2012, em parceira com a SMSS, para fortalecer a integração ensino-serviço-comunidade e qualificar as práticas de formação e cuidado integral em rede, orientadas pelas necessidades de saúde da população de maior vulnerabilidade social.

O projeto pedagógico do campus Baixada Santista tem garantido a docentes, alunos, técnicos e parceiros um dinâmico modo de reflexão e construção do processo ensino-aprendizagem.

Foram apresentadas algumas experiências de formação profissional relacionadas aos diferentes contextos onde se situam e ao tempo que exigiram para sua maturação.

A experiência da USP-SP ocorreu articulada às mudanças no panorama sociopolítico e sanitário do Brasil, nas quais a participação dos docentes e profissionais, por meio dos movimentos sociais para a formulação das políticas públicas, foi crucial para a criação de propostas e serviços que, de fato, podem transformar a lógica de atenção à saúde nos diferentes grupos populacionais relacionada à prática do terapeuta ocupacional.

As alternativas de formação apresentadas pela UFSCar, EBMSP e UNIFESP-BS se desenvolveram engajadas nesse movimento que já rendia frutos. Os profissionais, docentes e estudantes vivenciaram essas experiências inovadoras e puderam compartilhar esse processo de construção de diferentes perspectivas de formação profissional para o SUS nesta última década.

\section{Considerações finais}

A terapia ocupacional é uma profissão que contribui para a compreensão da complexidade dos problemas de saúde da populaçáo e tem trabalhado seu instrumental teórico e metodológico para desenvolver estratégias assistenciais compatíveis com os desafios colocados pela atenção à saúde no SUS, além de estar engajada nos movimentos que visam à institucionalização das articulaçóes ensino-serviço para maior qualificação de seus profissionais.

Este texto buscou dialogar com as principais questôes colocadas no grupo de trabalho sobre formação profissional do I Simpósio em APS e com as experiências de cursos de Terapia Ocupacional.

Nesse sentido, considera-se fundamental conhecer mais profundamente as diferentes experiências de formação profissional na APS dos cursos do país e engajar-se no seu fortalecimento, independentemente das políticas indutoras. Ao mesmo tempo em que é necessário sensibilizar os profissionais em atividade a 
refletirem sobre sua prática profissional nas unidades de saúde, que contam ou não com Estratégia de Saúde da Família, como também nos Núcleos de Apoio à Saúde da Família, estratégia e diretriz assistencial inovadora e que demanda tanto a formação de profissionais como estudos e pesquisas sobre seu alcance e importância para o estabelecimento da universalização, atenção integral e equidade no SUS.

As experiências e indagaçóes trazidas pelo desenvolvimento histórico da articulação entre cursos de Terapia Ocupacional, universidades, serviços, comunidades e movimentos sociais têm contribuído e podem ser potentes para a formação de docentes e de profissionais comprometidos com a construção de alternativas de cuidado em serviços de APS e de outros níveis assistenciais, para o aperfeiçoamento do SUS. Ainda são muitos os desafios colocados tanto para melhor qualificar esse ensino e integração como para realizar na prática assistencial a radical universalização da atenção e o exercício de direitos da população acompanhada em terapia ocupacional. Os profissionais, estudantes e docentes têm demonstrado interesse e engajamento na continuidade desse debate.

\section{Agradecimentos}

Nossos agradecimentos às colegas da Universidade de São Paulo Eucenir Fredini Rocha, Maria Helena Morgani de Almeida e Marta Carvalho de Almeida e à colega da Universidade Federal de Sáo Carlos Roseli Esquerdo Lopes, pelas contribuições na recuperação de informaçôes e da memória de algumas atividades relacionadas às experiências institucionais.

\section{Referências}

AlmeidA, M. C.; OLIVER, F. C. Abordagens comunitárias e territoriais em reabilitação de pessoas com deficiências: fundamentos para a Terapia Ocupacional. In: DE CARLO, M. M. R. P.; BARTALOTTI, C. C. (Orgs.). Terapia Ocupacional no Brasil: fundamentos e perspectivas. São Paulo: Plexus, 2001. p. 81-98.

ALMEIDA, M. C.; TISSI, M. C.; OLIVER, F. C. Deficiência e Atenção Primária em Saúde: do conhecimento à invenção. Revista de Terapia Ocupacional da Universidade de São Paulo, Sáo Paulo, v. 11, n. 1, p. 35-44, jan./abr. 2000. ANTUNES, M. H.; ROCHA, E. F. Desbravando novos territórios: incorporação da Terapia Ocupacional na estratégia da saúde da família no município de São Paulo e a sua atuação na atenção à saúde da pessoa com deficiência - no período de 2000-2006. Revista de Terapia Ocupacional da Universidade de São Paulo, São Paulo, v. 22, n. 3, p. 270-278, set./dez. 2011.

AOKI, M. Reabilitação com ênfase no Território: demandas de pessoas com deficiências e a promoção da participaçáo comunitária. 2009. 182 f. Dissertação (Mestrado em
Ciências da Reabilitação)-Universidade de São Paulo, Sáo Paulo, 2009.

BIANCHI, P. C.; MALFITANO, A. P. S. Terapia Ocupacional e açöes técnicas em contextos de vulnerabilidade social: distinçóes e proximidades entre o campo social e a área de atençáo básica em saúde. São Carlos: Universidade Federal de São Carlos, 2011. Relatório de Iniciação Científica.

BRASIL. Ministério da Saúde. Departamento de Atenção Básica. Guia prático do programa Saúde da Família. Brasília: Ministério da Saúde, 2001.

BRASIL. Ministério da Educação. Resolução CNE/CES $n^{\circ}$ 6, de 19 de fevereiro de 2002. Institui as Diretrizes Curriculares Nacionais para cursos de graduação em Terapia Ocupacional. Diário Oficial da República Federativa do Brasil, Poder Executivo, Brasília, DF, 04 mar. 2002a. Seção 1, p. 12. Disponível em: <http://portal.mec.gov. br/cne/arquivos/pdf/CES062002.pdf>. Acesso em: 28 out. 2010.

BRASIL. Ministério da Saúde. Ministério da Educação. Organização Pan-americana da Saúde. PROMED: Programa de Incentivo a Mudanças Curriculares nos Cursos de Medicina. Brasília: Secretaria de Políticas da Saúde, 2002b.

BRASIL. Ministério da Saúde. Ministério da Educação. Programa Nacional de Reorientação da Formação Profissional em Saúde - PRO Saúde. Brasília: Secretaria de Políticas da Saúde, 2007a.

BRASIL. Ministério da Saúde. Ministério da Educação. Portaria Interministerial no 3.019, de 26 de novembro de 2007. Dispóe sobre o Programa Nacional de Reorientação da Formação Profissional em Saúde. Diário Oficial da República Federativa do Brasil, Poder Executivo, Brasília, DF, 27 nov. 2007b. Seção 1. Disponível em: <http://portal.saude.gov.br/portal/arquivos/pdf/ portaria_3019.pdf >. Acesso em: 30 jul. 2012.

BRASIL. Ministério da Saúde. Portaria GM no 154 de 24 de janeiro de 2008. Cria os Núcleos de Apoio à Saúde da Família - NASF. Diário Oficial da República Federativa do Brasil, Poder Executivo, Brasília, DF, 25 jan. 2008a. Seção 1. Disponível em: <http://189.28.128.100/dab/ docs/legislacao/portaria154_24_01_08.pdf>. Acesso em: 18 maio 2012 .

BRASIL. Ministério da Saúde. Ministério da Educação. Portaria Interministerial no 1.802 , de 26 de agosto de 2008. Institui o Programa de Educação pelo Trabalho para a Saúde. Diário Oficial da República Federativa do Brasil, Poder Executivo, Brasília, DF, 27 ago. 2008b. Seção 1, p. 27. Disponível em: <http://portal.saude.gov.br/portal/ arquivos/pdf/portariainterm1802260808.pdf>. Acesso em: 31 jul. 2012.

BRASIL. Ministério da Saúde. Ministério da Educação. Portaria Interministerial no 421, de 03 de março de 2010. Dispóe sobre o Programa de Educação pelo Trabalho para a Saúde uma das estratégias do PRO Saúde. Diário Oficial da República Federativa do Brasil, Poder Executivo, Brasília, DF, 05 mar 2010. Seção 1, n. 43, p. 52-53.

BRASIL. Ministério da Saúde. Programa de Reorientação da Formação Profissional em Saúde - PRO Saúde dados 2011. Brasília: Ministério da Saúde, 2011a. Cursos (folder). 
Disponível em: <http:/www.prosaude.org/noticias/ sem2011Pro/index.php>. Acesso em: 08 maio 2012.

BRASIL. Ministério da Saúde. Portaria no 2.488, de 21 de outubro de 2011. Dispóe sobre a Política Nacional de Atenção Básica. Diário Oficial da República Federativa do Brasil, Poder Executivo, Brasília, DF, 24 out. 2011b. Seção 1. Disponível em: <http://www.brasilsus.com. br/legislacoes/gm/110154-2488.html>. Acesso em: 18 maio 2012.

BRASIL. Ministério da Saúde. Abrangência Nacional do Programa PET-Saúde. Brasília: Ministério da Saúde, 2012. Disponível em: <http://portal.saude.gov.br/portal/saude/ profissional/area.cfm?id_area $=1597>$. Acesso em: 19 maio 2012.

CALDEIRA, V. A. Prática de terapia ocupacional em unidade básica de saúde na atenção às pessoas com deficiência. 2009. 170 f. Dissertação (Mestrado em Ciências da Reabilitação)-Universidade de São Paulo, São Paulo, 2009.

CAMPOS, G. W. S. Reflexões sobre a clínica ampliada em equipes de saúde da família. In: CAMPOS, G. W. S. Saúde Paidéia. São Paulo: Hucitec, 2003. p. 68-77.

CARVALHO, V. C.; MATSUKURA, T. S. Identificando práticas de terapeutas ocupacionais inseridos no contexto da atenção básica de saúde. São Carlos: Universidade Federal de São Carlos, 2011. Relatório de Iniciação Científica.

CARRASCO-BASSI, B. G. C. Terapia Ocupacional na Atenção Básica em Saúde no município de São Carlos: um enfoque nas pessoas com deficiência e nas pessoas em sofrimento mental. 2012. 134 f. Dissertação (Mestrado em Terapia Ocupacional)-Universidade Federal de São Carlos, São Carlos, 2012.

CECCIM, R. B.; CARVALHO, Y. M. Ensino da saúde como projeto da integralidade: a educaçáo dos profissionais de saúde no SUS. In: PINHEIRO, R.; CECCIM, R. B.; MATTOS, R. A. (Orgs.). Ensinar saúde: a integralidade e o SUS nos cursos de graduação na área da saúde. Rio de Janeiro: IMS/UERJ; CEPESQ; ABRASCO, 2005. p. 69-92.

CECCIM, R. B. A emergência da educação e ensino da saúde: interseçôes e intersetorialidades. Revista Ciência \&. Saúde, Porto Alegre, v. 1, n. 1, p. 9-23, jan./jun. 2008.

CONSELHO FEDERAL DE FISIOTERAPIA E TERAPIA OCUPACIONAL - COFFITO. Oficina "Equidade, ética e direito à saúde: formação e ação da Terapia Ocupacional”. In: CONGRESSO BRASILEIRO DE CIÊNCIAS SOCIAIS E HUMANAS EM SAÚDE, 5., CONGRESSO DA ASSOCIAÇÃO LATINO AMERICANA DE MEDICINA SOCIAL, 10., CONGRESSO DA ASSOCIAÇÃO INTERNACIONAL DE POLÍTICAS DE SAÚDE, 14., 2007, Salvador, Bahia. Anais... Salvador: COFFITO, 2007. Relatório das atividades.

CRUZ, D. M. C.; NICOLAU, S. M.; FARIA, C. B. Tecnologia Assistiva de baixo custo para pessoas com deficiências no território: ampliando a independência e as possibilidades de participação social. In: OLIVEIRA, A. I. A. (Org). Inovaçâo Tecnológica e Inclusão Social. Belém: EdUEPA, 2011. p. 141-150.
FEUERWERKER, L. Educação dos profissionais de saúde hoje - problemas, desafios, perspectivas e as propostas do Ministério da Saúde. Revista ABENO, São Paulo, v. 3, n. 1, p. 24-27, 2003

FERrEIRA, T. G. Pessoas com deficiência: condiçôes de convivência e possibilidades de atenção domiciliar. 2009 98 f. Dissertação (Mestrado em Ciências da Reabilitação)Universidade de São Paulo, São Paulo, 2009.

GIL, C. R. R. Atenção primária, atenção básica e saúde da família: sinergias e singularidades do contexto brasileiro. Cadernos de Saúde Pública, Rio de Janeiro, v. 22, n. 6, p. 1171-1181, jun. 2006.

HADDAD, A. E. A enfermagem e a Política Nacional de Formação dos Profissionais de Saúde para o SUS. Revista da Escola de Enfermagem da USP, São Paulo, v. 51, n. 3, p. 1803-09, 2011. http://dx.doi.org/10.1590/ S0080-62342011000800029

HADDAD, A. E. et al. Formação de profissionais de saúde no Brasil: uma análise no período de 1991 a 2008. Revista de Saúde Pública, São Paulo, v. 44, n. 3, p. 5-9, maio 2010. http://dx.doi.org/10.1590/S0034-89102010005000015 HO, D. C.; OLIVER, F. C. Terapia ocupacional e saúde da pessoa com deficiência na Secretaria Municipal de Saúde: uma discussão sobre dez anos de sua incorporação. Revista de Terapia Ocupacional da Universidade de São Paulo, São Paulo, v. 16, n. 3, p. 107-116, set./dez. 2005. LANCMAN, S.; BARROS, J. O. Estratégia de saúde da família (ESF), Núcleo de Apoio à Saúde da Família (NASF) e terapia ocupacional: problematizando as interfaces. Revista de Terapia Ocupacional da Universidade de São Paulo, São Paulo, v. 22, n. 3, p. 262-269, dez. 2011. LOPES, R. E. Cidadania, Politicas Públicas e Terapia Ocupacional, no contexto das açóes de saúde mental e saúde da pessoa portadora de deficiência, no município de São Paulo. 1999.548 f. Tese (Doutorado em Educação)Universidade Estadual de Campinas, Campinas, 1999. LOPES, R. E.; PALMA, A. M. Possibilidades e limites da terapia ocupacional no Programa de Saúde da Família - PSF. São Carlos: Laboratório METUIA, Pró-Reitoria de Pesquisa da Universidade Federal de São Carlos, 2005. Relatório de Pesquisa.

LOPES, R. E.; FURLAN, P. G.; BRITO, E. M. Ações de Saúde Básica: o caso do Município de São Carlos. São Carlos: Laboratório METUIA; Universidade Federal de São Carlos; CNPq, 2006. Relatório de Pesquisa.

LOPES, R. E. et al. Açôes básicas de saúde e a construção do SUS: cidadania, direitos e políticas públicas. São Carlos: Laboratório METUIA; Universidade Federal de São Carlos; CNPq; Ministério da Saúde, 2007. Relatório de Pesquisa.

MARSIGLIA, R. G. Relação Ensino/Serviços: dez anos de Integração Docente Assistencial (IDA) no Brasil. São Paulo: HUCITEC, 1995.

MELLO, G. A.; FONTANELLA, B. J. B.; PIVA, M. M. Atenção Básica e Atenção Primária em Saúde - origens e diferenças conceituais. Revista de APS, Juiz de Fora, v. 12, n. 2, p. 204-213, abr./jun. 2009.

MENDES, E. V. Uma agenda para a saúde. São Paulo: Hucitec, 1996. 
MERHY, E. E Um ensaio sobre o médico e suas valises tecnológicas. Interface: Comunicacao, Saude, Educacao, Botucatu, v. 6, n. 6, p. 109-125, fev. 2000. http://dx.doi. org/10.1590/S1414-32832000000100009

MERHY, E. E.; FRANCO, T. B. Mapas analiticos: um olhar sobre a organização e seus processos de trabalho. Rio de Janeiro: UFRJ, 2006. Disponível em: <http://www.medicina.ufrj.br/micropolitica/pesquisas/ atencaodomiciliar/textos/mapas analíticos.pdf $>$. Acesso em: 30 maio 2012.

NICOLAU, S. M.; AOKI, M.; OLIVER, F. C. A atençâo às pessoas com deficiências no território: uma experiência da Terapia Ocupacional no Programa de Saúde da Família em um bairro periférico da cidade de Sáo Paulo. Revista de Terapia Ocupacional da Bahiana, Salvador, v. 3, n. 1, p. 5-10, 2007.

NUNES, A. C.; DELLA BARBA, P. C. S. Identificação de crianças com deficiências na regiāo do Jardim Jóquei Clubel Guanabara por meio da Estimativa Rápida Participativa e proposta de intervenção em Terapia Ocupacional. São Carlos: Universidade Federal de São Carlos, 2010. Relatório de Projeto de Extensão.

OLIVEIRA, G. N. O projeto terapêutico e a mudança nos modos de produzir saúde. São Paulo: Hucitec, 2008.

OLIVER, F. C. et al. Reabilitação baseada no Território: construindo a participação na vida social. Revista de Terapia Ocupacional da Universidade de São Paulo, São Paulo, v. 12, n. 1-3, p. 8-14, jan./dez. 2001.

OLIVER, F. C.; BARROS, D. D.; LOPES, R. E. Estudo sobre a incorporação da terapia ocupacional no contexto das ações de saúde mental e saúde da pessoa com deficiência no Município de São Paulo entre 1989 e 1993. Revista de Terapia Ocupacional da Universidade de São Paulo, São Paulo, v. 16, n. 1, p. 31-39, jan./abr. 2005.

OLIVER, F. C. et al. Terapia ocupacional na Atenção Primária em Saúde: contribuiçóes ao estudo do percurso de ensino e pesquisa no Curso de Terapia Ocupacional da Faculdade de Medicina da USP. 2012. 10 p. Mimeo.

PALM, R. C. M. Catálogo Latinoamericano de Asociaciones, Carreras y Postgrados de Terapia Ocupacional. Curitiba: RENETO, 2012.

PIMENTEL, A. M.; COSTA, M. T. B.; SOUZA, F. R. Terapia Ocupacional na Atenção Básica: a construção de uma prática. Revista de Terapia Ocupacional da Universidade de São Paulo, São Paulo, v. 22, n. 2, p. 110-116, maio/ ago. 2011.

PIMENTEL, A. M.; OLIVER, F. C.; UCHÔAFIGUEIREDO, L. Formação do Terapeuta Ocupacional para o Trabalho na Atenção Primária em Saúde (APS). In: SEMINÁRIO DE TERAPIA OCUPACIONAL NA APS, 1., 2012, São Paulo. São Paulo: [s.n.], 2012. 4 p. Mimeo.
REDE UNIDA. Nossa História Linha do Tempo. Disponível em: <http://www.redeunida.org.br/rede-unida/ nossa-historia>. Acesso em: 10 maio 2012.

ROCHA, E. F.; SOUZA, C. C. B. X. Terapia ocupacional em reabilitação na atenção primária à saúde: possibilidades e desafios. Revista de Terapia Ocupacional da Universidade de São Paulo, Sáo Paulo, v. 22, n. 1, p. 36-44, jan./abr. 2011. ROCHA, E. F.; KRETZER, M. R. Ações de reabilitação de pessoas com deficiência na estratégia da saúde da família da Fundação Zerbini e Secretaria Municipal de Saúde de São Paulo - Região Sudeste - Sapopemba/ Vila Prudente - período 2000/2006. Revista de Terapia Ocupacional da Universidade de São Paulo, São Paulo, v. 20, n. 1, p. 59-67, abr. 2009.

ROCHA, E. F.; PAULA, A. R.; KRETZER, M. R. O estudo de prevalência de deficiências e incapacidades como instrumento de planejamento das atividades de atenção à saúde e reabilitação no Programa Saúde da Família. Revista de Terapia Ocupacional da Universidade de São Paulo, São Paulo, v. 15, n. 1, p. 1-10, abr. 2004.

SILVEIRA, R.; BRITO, C. M. D. O clown como recurso renovável de promoçáo de saúde no território. In: CONGRESSO BRASILEIRO DE TERAPIA OCUPACIONAL, 12.; CONGRESSO LATINO AMERICANO DE TERAPIA OCUPACIONAL, 9., 2011, São Paulo. Cadernos de Terapia Ocupacional da UFSCar, São Carlos, v. 19, 2011. Suplemento Especial. CD-ROM. SOARES, L. B. T. A Terapia Ocupacional na Atenção Básica no Município de São Carlos, São Paulo: a Residência Multiprofissional em Saúde da Família e Comunidade da UFSCar antecipa os núcleos de Apóio em Saúde da Família. Revista de Terapia Ocupacional da Universidade de São Paulo, São Paulo, v. 19, p. 41-42, 2008. Suplemento 1. UNIVERSIDADE FEDERAL DE SÃO CARLOS - UFSCar. Centro de Ciências Biológicas e da Saúde. Departamento de Terapia Ocupacional. Coordenação do Curso de Graduação em Terapia Ocupacional da UFSCar. Projeto Pedagógico do Curso de Terapia Ocupacional. São Carlos: UFSCar, 2007. 84 p. UNIVERSIDADE FEDERAL DE SÁO PAULO - UNIFESP. Campus Baixada Santista. $A$ educação interprofissional na formação em saúde: a competência para o trabalho em equipe e para a integralidade no cuidado. Santos: UNIFESP, 2006. Projeto Político Pedagógico.

VARELA, R. C. B. Crianças com deficiência: compreendendo seu cotidiano e a importância do uso de recursos tecnológicos na atenção em terapia ocupacional. 2010. 212 f. Dissertação (Mestrado em Ciências da Reabilitação)-Faculdade de Medicina, Universidade de São Paulo, São Paulo, 2010.

\section{Contribuição dos Autores}

Todas as autoras foram responsáveis pela concepção e redação do presente manuscrito, assim como pela revisão e aprovação final para publicação. 